\title{
A cross-phase reaction coordinate in the formation of a mononuclear copper(II) orotate complex
}

\author{
L Falvello ${ }^{1}$, Z Basdouri' ${ }^{2}$, M Graia ${ }^{3}$, M Tomás ${ }^{4}$ \\ ${ }^{1}$ Dept of Inorganic Chemistry, Univ of Zaragoza, Zaragoza, ${ }^{2}$ Department of Inorganic Chemistry \\ and Aragón Materials Science Institute (ICMA), University of Zarag, Zaragoza, Spain, ${ }^{3}$ Laboratoire \\ de Matériaux, Cristallochimie et Thermodynamique Appliquée, Département de Chimie and Un, \\ Tunis and Sfax, Tunisia, ${ }^{4}$ Department of Inorganic Chemistry and Institute for Chemical Synthesis \\ and Homogeneous Catalysis (IS, Zaragoza, Spain \\ falvello@unizar.es
}

The reaction of orotic acid with a copper (II) starting material in the presence of $\mathrm{CsOH}$ produces crystals of a sixcoordinate Jahn-Teller active $\mathrm{Cu}$ complex (1, Fig. 1). The initial solid product loses crystallinity when removed from its aqueous mother liquor. When crystals of (1) are kept in the mother liquid, and the aqueous supernatant is partially evaporated, they evolve to a second anionic mononuclear copper complex, this one four-coordinate, in a solvent-mediated crystal-to-crystal transformation. The crystal structure of this second copper complex (2, Fig. 2) is conserved both within and without the mother liquor. A previously reported nickel complex (3), isostructural to (1), is stable at ambient conditions both in the presence and in the absence of mother liquor. Comparisons of the molecular and extended structures, along with TGA data, were used in an attempt to elucidate the comparative stabilities of the isostructural $\mathrm{Cu}(\mathbf{1})$ and $\mathrm{Ni}(3)$ complexes in their respective solids, and the role of the relative stability of (2) in promoting the further reaction of (1) after its initial formation. An unusual feature in the anisotropic displacement parameters from the structure analysis of compound (2) is briefly discussed. [1] Using the crystal to engineer the molecule: cis-trans-isomer selection in anionic bis(orotate) complexes. Larry R. Falvello, Daniel Ferrer, María Piedrafita, Tatiana Soler and Milagros Tomás. CrystEngComm, 2007, 9, 852-855. FUNDING: Ministerio de Ciencia e Innovación (Spain, Grant PGC2018-093451-B-I00), the European Union Regional Development Fund, FEDER), and the Diputación General de Aragón, Project M4, E11_20R.

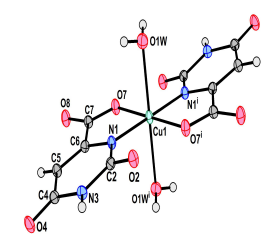

Figure 1

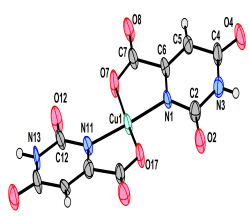

Figure 2

Acta Cryst. (2020). A76, a167 